\title{
Appendix 2: Content of Educational Programs in Allergy and Allergic Asthma
}

\section{Allergic Asthma and Allergic Rhinitis}

Appropriate actions for guided self-management include

1 Developing good communication between patient and physician to improve patient compliance

2 Promoting understanding about the basic facts, causes and triggers of allergic asthma/allergic rhinitis

3 Identifying and controlling factors that aggravate asthma/rhinitis symptoms and provoke exacerbations

4 Following a written action plan to avoid or handle exacerbations

5 Emphasizing the importance of proper drug use and correct use of spacers and inhalers for long-term control

6 Monitoring symptoms and peak flow values in persistent asthma and adjusting medication accordingly

7 Educating to decrease reliance on unproven treatments

\section{Eczema}

\section{Educational programs in eczema should include}

1 Information about the nature, heredity, causes and triggers of eczema

2 Identification and avoidance of individual provocation factors, skin care and treatment options, including complementary therapies

3 Discussion of diagnosis and treatment of food allergies and adequate nutrition in childhood behaviour-oriented psychological intervention to interrupt the itching-scratching cycle

4 Training to improve stress-management and reduce the negative social effects of illness-specific problems 


\section{Severe Reactions, Allergic Anaphylaxis}

Each patient should have an individual management protocol to include

1 Carrying an epinephrine auto-injector and knowing how and when to use it; always have a spare auto-injector available

2 Carrying an emergency pager

3 Carrying/wearing MedicAlert information

4 Avoiding stinging insects, learning how not to attract them

5 Avoiding allergenic ingredients in ready-made food

In the school environment

6 Education of parents, teachers, fellow students, and school administrators is necessary to provide a safe environment for children at risk for anaphylaxis to foods or insect stings

7 Creating a no-food area of the school playground

8 Identifying a supervisor to carry a telephone for emergencies

In the occupational environment

9 Health and safety measures should be introduced to prevent exposure of affected workers to airborne or contact allergens 